\title{
An Unternehmenswerten und politischem Rahmen ansetzen
}

\author{
Die Sustainable Balanced Scorecard verspricht mehr Nachhaltigkeit in der Un- \\ ternehmensführung. Für ihre maximale Wirkung muss die gesamte Strategie \\ eines Unternehmens mit Hilfe der Scorecard optimiert werden. Ein ebenso \\ wichtiger Erfolgsfaktor sind besonders für kleine und mittlere Unternehmen \\ langfristig stabile Rahmenbedingungen.
}

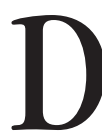
ie systematische Integration von Nachhaltigkeitserfordernissen in die Unternehmensfïhrung wird bislang von kaum einem Unternehmen geleistet. Daher wird in jüngster Zeit in einigen Projekten versucht, das gebräuchliche ManagementInstrument Balanced Scorecard um die Nachhaltigkeitsdimension zu erweitern (1). Das RKW Hessen führte von 2001 bis 2003 das Forschungsprojekt "Sustainable Balanced Scorecard" (SBS) durch, welches vom Bundesministerium für Bildung und Forschung gefördert wurde (2). Ziel des Projekts war zum einen zu klären ob und inwieweit es möglich ist, Nachhaltigkeitsaspekte mit dem Instrument der Balanced Scorecard zu implementieren und andererseits zu überprüfen, ob und in welcher Form die SBS auch ein geeignetes Instrument für mittelständische Unternehmen (KMU) ist.

\section{Ohne Strategie keine Nachhaltigkeit}

Nachhaltige Entwicklung fordert eine Abkehr von kurzfristigem Gewinnstreben zugunsten von längerfristigen Entwicklungspfaden. Die Orientierung an Langfristigkeit unterstreicht die wichtigste Voraussetzung für die Erarbeitung einer Sustainable Balanced Scorecard: Eine ausformulierte, an ökonomischer, ökologischer und sozialer Nachhaltigkeit orientierte Strategie. Entscheidend für die erfolgreiche Formulierung einer Strategie ist, so ein wichtiges Ergebnis des Forschungsprojekts, die generellen Werte der Unternehmenseigner und das Unternehmensleitbild in den Strategieprozess einzubeziehen.

Bei der Strategieentwicklung wird für das gesamte Unternehmen und, wenn relevant, für einzelne Geschäftsfelder eine strategische Ausgangsposition bestimmt:
- Im Rahmen der Umfeldanalyse werden Chancen und Risiken für das Unternehmen verortet und Signale, die auf eine Veränderung im Geschätsumfeld deuten, analysiert. Dazu gehören politische, rechtliche, wirtschaftliche, ökologische, soziale und technologische Entwicklungen im unternehmerischen Wirkungsfeld die per se Nachhaltigkeitsbeziige aufweisen.

- Bei der Unternehmens- oder Potenzialanalyse werden die Stärken und Schwächen des Unternehmens analysiert. Die Bewertung der Ressourcen und Kompetenzen zur Bewältigung zukünftiger Herausforderungen bestimmen entscheidend den Handlungswillen in Bezug auf die Verankerung von Nachhaltigkeitsaspekten.

Im Anschluss an die Analyse der Ausgangsposition werden die Unternebmens- bzw. Geschäftsfeldstrategien formuliert. Unternehmensstrategien umfassen Entscheidungen mit Grundsatzcharakter. Hier wird die Handlungsbereitschaft hinsichtlich nachhaltigen Wirtschaftens fixiert. Es hat sich deutlich gezeigt, dass bei der Strategieformulierung die persönlichen Einstellungen und Werthaltungen der Inhaber einen hohen Einfluss haben und inhaltlich weit über eine Ertragsorientierung hinausgehen.

\section{Einführung der SBS in KMU}

Die Balanced Scorecard ist ein Instrument das eingesetzt wird, um

- Strategien schrittweise zu konkretisieren,

- Unternehmensstrategien und Ziele zu kommunizieren,

- Strategien mit der konkreten Jahresplanung und Budgetierung zu verknüpfen und

- Fortschritte zu messen.

Die am Forschungsprojekt beteiligten mittelständischen Unternehmen erwarteten darüber hinaus, dass an Aspekten der Nachhaltigkeit orientierte Strategien besser konkretisiert werden können und Führungskennzahlen zur Messung ökologischer und sozialer Ziele sich problemlos ins Berichtswesen integrieren lassen.

Die Ausgewogenheit der Ziele wird für die wesentlichen Handlungsebenen oder Perspektiven eines Unternehmens hergestellt. Die gängigen vier Perspektiven reichen zur Verankerung des Nachhaltigkeitsgedankens für die meisten mittelständischen Unternehmen aus. Es wurde im Projekt bewusst auf eine zusätzliche Nachhaltigkeitsperspektive verzichtet, um zu vermeiden, dass ein Auffangbecken für alles, was in eine "richtige" Balanced Scorecard nicht passt, geschaffen wird.

In der Finanzperspektive ist der ökonomische Bezug eindeutig und wird allenfalls durch die Zielsetzungen der anderen Perspektiven in seiner wertmäßigen Ausprägung relativiert.

In der Kundenperspektive werden Zielsetzungen bezüglich Zielgruppe, Marktpositionierung und Marktauftritt getroffen. Ökologische und soziale Nachhaltigkeitsbezïge werden in der Kundenperspektive verankert, wenn es zum Beispiel um die Etablierung eines besonderen Sortiments (Produkte mit Öko Label, fair trade etc.) geht oder die Gewinnung von nachhaltigkeitsbewussten Zielgruppen geplant ist.

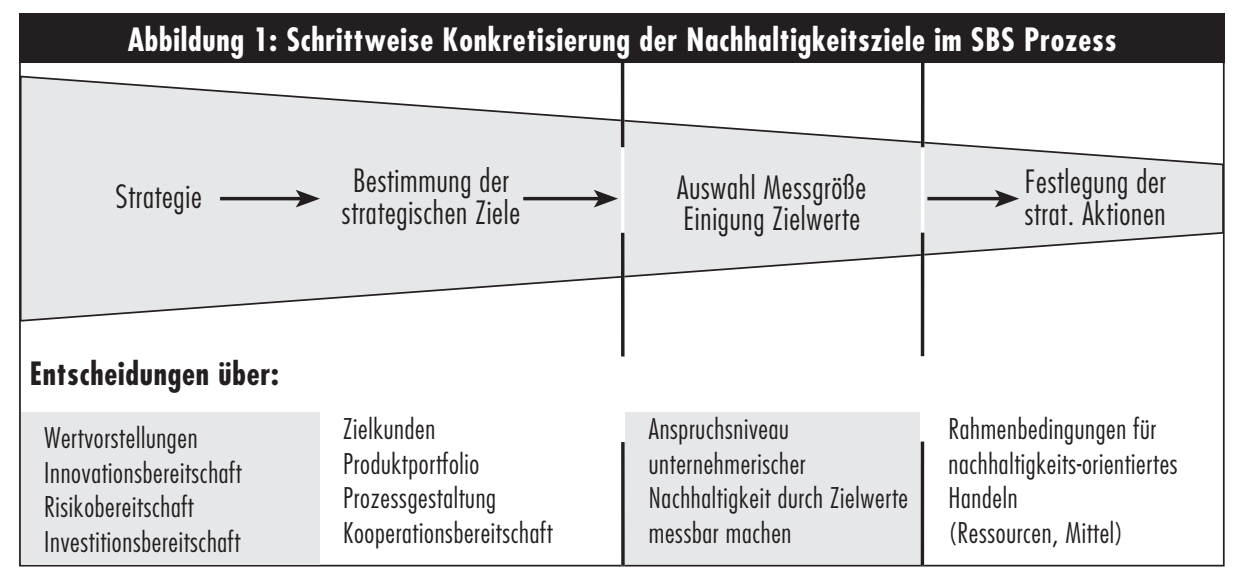




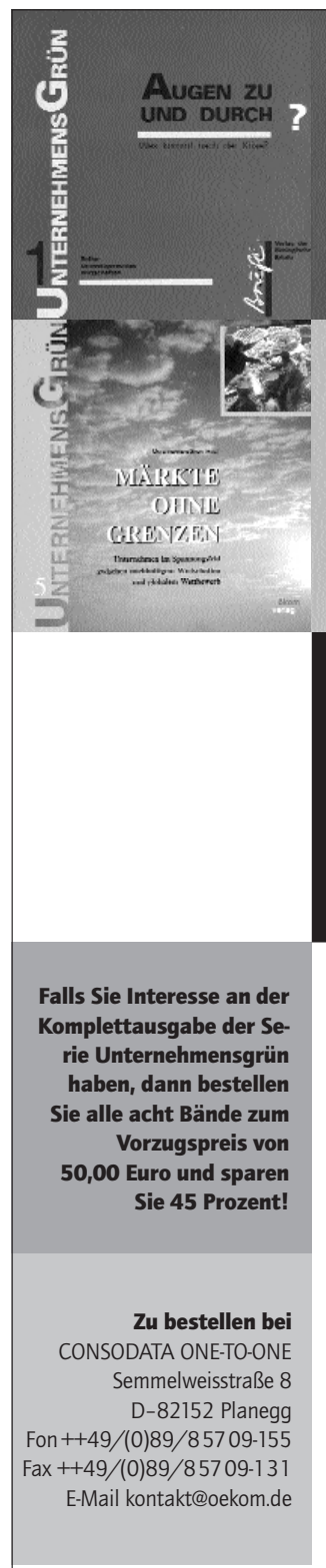

Immer wieder

Topaktuell!

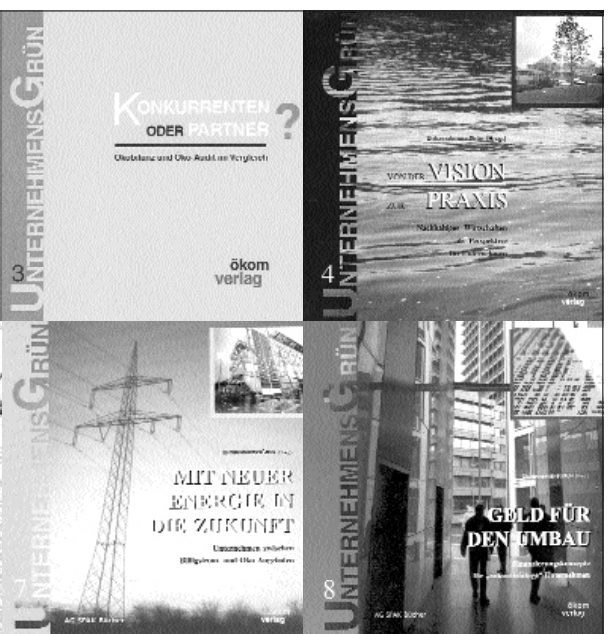

Die Serie Unternehmensgrün Umweltgerechtes Wirtschaften im ökom Verlag
Unternehmensgrün 5_Märkte ohne Grenzen. Unternehmen im Spannungsfeld zwischen nachhaltigem Wirtschaften und globalem Wettbewerb_ISBN 3-92824439-6 Preis: 10,10 Euro_Können Unternehmen international agieren und kooperieren ohne ihre soziale und ökologische unternehmerische Verantwortung zu vergessen? Sind Nachhaltigkeit und Globalisierung unvereinbare Gegensätze? Eröffnet die Globalisierung des Weltmarkts auch Chancen für kleine Unternehmen? Diesen und viele anderen Fragen gehen Autoren aus Wirtschaft und Wissenschaft in diesem Buch nach.+++ Unternehmensgrün 6__Arbeit: Strategien der Existenzsicherung_ISBN 3-928244-48-5 Preis: 10,10 Euro_In diesem Band werden verschiedene Strategien zur Vermeidung und Bekämpfung der Arbeitslosigkeit angesprochen. Ebenso wird ein Blick über den Tellerrand geworfen, wie andere Länder mit dem Abbau ihrer Arbeitslosigkeit umgehen. Mit diesen Themen beschäftigen sich Autoren aus Politik und Wirtschaft.+++ Unternehmensgrün 1_ Augen zu und durch? Was kommt nach der Krise? ISBN 3-92850705-2, Preis: 10,10 Euro+++ Unternehmensgrün 2_Erhalt durch Veränderung. Perspektiven einer ökologischen und sozialen Umsteuerung. ISBN 3-928244-13-2, Preis: 10,10 Euro+++ Unternehmensgrün 3_Konkurrenten oder Partner. Ökobilanz und ÖkoAudit im Vergleich. ISBN 3-928244-16-7, Preis: 10,10 Euro+++ Unternehmensgrün 4_Von der Vision zur Praxis. Nachhaltiges Wirtschaften als Perspektive für Unternehmen. ISBN 3-92824427-2, Preis: 15,30 Euro +++ Unternehmensgrün 7_Mit neuer Energie in die Zukunft. ISBN 3-928244-66-3, Preis: 12,80 Euro+++ Unternehmensgrün 8_Geld für den Umbau. ISBN 3-92824473-6, Preis: 12,80 Euro.
In der Prozessperspektive wird herausgearbeitet, welche Prozesse eine herausragende Bedeutung bei der Verwirklichung der Strategie haben und welche Prozesse etabliert werden müssen. Der Bezug zur ökologischen Nachhaltigkeit wird hier meist klassisch produktionsbezogen über Prozesseffizienz und Ausschussquoten hergestellt.

In der Potenzialperspektive wird aufgezeigt, auf welche zukünftigen Herausforderungen sich das Unternehmen einstellen muss. Dabei geht es zum Beispiel um die Entwicklung umweltverträglicherer Produkte, die Einführung neuer Technologien sowie um das Thema "Empowerment" und Eigenverantwortung der Mitarbeiter.

\section{Schrittweise Konkretisierung}

Die Konkretisierung der Strategie erfolgt schrittweise, indem strategische Handlungsoptionen in drei Stufen reduziert werden und in konkretes Projektmanagement münden. Dabei können auch strategisch wichtige Aspekte der Nachhaltigkeit verankert werden.

Strategische Ziele bestimmen: Die in Leitbild und Strategie formulierten Ansprüche an unternehmerische Nachhaltigkeit werden durch die Formulierung konkreter strategischer Ziele im Unternehmen verankert. Die mittelständischen Unternehmen im Projekt kamen mit deutlich weniger Zielen als üblicherweise beschrieben aus. Messgrößen auswäblen: Sind Ziele festgelegt und die wichtigsten Ursache-Wirkungszusammenhänge aufgezeigt, müssen Indikatoren für die Zielerreichung gefunden werden. Dabei muss die "Höhe der Erfolgslatte" für Nachhaltigkeitsaspekte bestimmt werden.

Strategische Aktionen festlegen: Die strategischen Ziele werden über strategische Aktionen umgesetzt. Die Ernsthaftigkeit mit der die einzelnen Ziele vorangebracht werden sollen, wird in diesem Schritt erkennbar. Dabei zeigte sich, dass ein funktionierendes Projektmanagement in kleinen und mittleren Unternehmen durchaus keine Selbstverständlichkeit ist.

Im Verlauf des Forschungsprojektes haben vier der fünf Pilotunternehmen eine SBS erarbeitet und implementiert. Ein Pilotunternehmen muss seine SBS noch fertigstellen.

\section{- Nachhaltigkeit durch die SBS?}

Die Sustainable Balanced Scorecard ist ein gut geeignetes Instrument zur Integration, Umsetzung und Kommunikation von nachhaltigen Unternehmenszielen, welches sich mit einer überschaubaren Anzahl von Schritten realisieren lässt. Das Bewusst- 
sein für Nachhaltigkeitszusammenhänge wird gefördert, indem ökologische und soziale Bezüge während des SBS Prozesses hinterfragt und integriert werden. Dabei hat sich die gewählte Vorgehensweise als eindeutig mittelstandstauglich bewiesen. Werden im Rahmen der Strategieentwicklung allerdings keine Entscheidungen zu Art und Umfang unternehmerischer Nachhaltigkeit getroffen, kann das Instrument SBS dieses Defizit nicht heilen.

\section{- Fazit: Kontextfaktoren stärken!}

Bei der Verankerung des Konzeptes auf Unternehmensebene stehen Eigentïmer und Geschäftsfiuhrer von kleinen und mittleren Unternehmen vor zwei Herausforderungen: Zum einen sollen sie einen konstruktiven Beitrag zur praktischen Umsetzung des politisch verwirrend formulierten Nachhaltigkeitsanspruchs leisten. Zum anderen erschwert die zunehmende Dynamisierung und Komplexität von Umfeldbedingungen die Verfolgung von Nachhaltigkeitszielen. Mittel- und langfristige Entwicklungen lassen sich aber umso besser verfolgen je klarer und stabiler die Rahmenbedingungen des Handelns sind. Daher ist es erforderlich, ein Anreizsystem zur Förderung unternehmerischer Nachhaltigkeit zu schaffen. Der auf politischer Ebene zu erzielende Grundkonsens umfasst

- Messbare Nachhaltigkeitsziele für Deutschland und die Europäische Union.

- Stabile Rahmenbedingungen der staatlichen und sozialen Subsysteme.

- Koordinierte und verständliche staatliche Förderpolitik, die über mehrere staatliche Haushaltsjahre verlässlich betrieben wird.

- Ein übersichtliches und auf Nachhaltigkeit bezogenes Umweltrecht.

Klarheit und Zuverlässigkeit bei den angestrebten politischen Zielen würde unmittelbar die Nachhaltigkeitsbestrebungen der Unternehmen befördern und Freiräume für unternehmerisches Handeln ermöglichen. Solange das nicht der Fall ist, reduziert sich die Einbeziehung von Nachhaltigkeitsaspekten auf die Frage persönlicher Werthaltungen des Unternehmers. Instrumente zur Realisierung einer nachhaltigen Unternehmensführung sind hinreichend vorhanden. Nun gilt es, die Rahmensetzungen für mehr Nachhaltigkeit zu verstärken.

\section{Anmerkungen}

(1) Vgl. Gminder, C.U. et al.: Nachhaltig managen mit der Balanced Scorecard. Erfahrungen aus einem Praxisprojekt. In: Ökologisches Wirtschaften Nr. 6/2002, S. 27-28.

(2) Informationen zum Projekt und den beteiligten Projektpartnern im Web unter www.rkw-hessen.de; die Autorinnen haben darin einzelbetriebliche Beratungen bei den Pilotunternehmen durchführt und eine Gruppe von 15 Unternehmen in einem Transferkreis fachlich betreut.

\section{Die Autorinnen}

Christel Geiger ist als Unternehmensberaterin tätig. Kontakt: Controlling Service Geiger, Friedberg , E-Mail: Geiger@c-s-geiger.de

Silke Hermann ist als Unternehmensberaterin tätig. Kontakt: Systemische Managementberatung Silke Hermann, Nussbaumstr. 8a, 65719 Hofheim a.T, Tel. 06192-952815,

E-Mail: Hermann@sysmanagement.de

\section{Aller Anfang ist schwer}

\section{Tourismus ist einer der dynamischsten und ökonomisch bedeutendsten globalen Wirtschaftszweige. Auch der Ökotourismus wächst - allerdings von einem klei- nen Niveau aus. Für Schritte aus der Nische bedarf es überzeugender Konzepte, die vorhandenen theoretischen Modelle auch erfolgreich in die Praxis umzuset- zen. Dies wird am Beispiel Lateinamerika illustriert.}

$\mathrm{T}$ Von Sonja Bartelt ourismus ist für viele Länder ein Hoffnungsträger der wirtschaftlichen Entwicklung. Er ist eine wichtige Devisenquelle und schafft Arbeitsplätze. Kein anderer Wirtschaftszweig hat nach Angaben der Welttourismusorganisation (WTO) von letztem Jahr Zuwachsraten von bis zu 1010 Prozent zu verzeichnen. Auf Entwicklungsländer, einschließlich der Schwellenländer, entfällt mit deutlich über 100 Milliarden US Dollar knapp ein Viertel der damit erzielten Deviseneinnahmen.

In den Zielländern führt die expansive Ausrichtung auf den Ausbau von touristischen Einrichtungen allerdings zu einer Überbeanspruchung der natürlichen Ressourcen und somit häufig zum Verlust von biologischer Vielfalt sowie der
Beeinträchtigung von sozialen und kulturellen Strukturen. Wie kaum eine andere Branche ist Tourismus jedoch auf eine intakte Natur und Umwelt angewiesen. Um einen positiven Beitrag zu einer nachhaltigen Entwicklung zu leisten, muss der Tourismus soziale, ökologische, kulturelle und wirtschaftliche Verträglichkeitskriterien erfüllen.

Trotz seiner Bedeutung wurde ihm jedoch in der internationalen Nachhaltigkeits- und Naturschutz-diskussion lange Zeit keine Beachtung geschenkt. Sowohl in der Agenda 21, der Klimarahmenkonvention als auch in der Biodiversitätskonvention wird Tourismus lediglich am Rande erwähnt.

Obwohl auch die Ökotourismus-Branche derzeit wächst, sind die Umsätze verglichen mit denen des Massentourismus eher gering. Laut Umweltprogramm der Vereinten Nationen (UNEP) liegt der Marktanteil bei drei bis fünf Prozent, was immerhin drei bis fünf Milliarden US Dollar Einnahmen aus Ökotourismus in den Entwicklungsländern bedeutet.

Durch einen langjährigen Missbrauch des Begriffs Ökotourismus wurde jedoch vielen Endverbrauchern die Sicherheit genommen, dass die Nutzung von Ökotourismuseinrichtungen zum Erhalt von Naturschutzgebieten oder der Biodiversität im Zielland beiträgt. Probleme wie die Schwierigkeit der Qualitätskontrolle oder ein fehlendes Verständnis des Begriffs Ökotourismus als solchen wurden letztes Jahr zum ersten Mal auf internationaler Ebene aufgegriffen. Die bei den Vereinten Nationen angesiedelte Kommission für Nachhaltige Entwicklung erklärte das Jahr 2002 zum internationalen Jahr des Ökotourismus.

Im Rahmen des UNEP wurden zusammen mit der WTO regionale Vorbereitungstreffen in der ganzen Welt organisiert, in denen Nichtregierungsorganisationen, staatliche Organisationen und Vertreter aus Wirtschaft und der Wissenschaft zusammenkamen, um globale Problemlagen zu diskutieren. Die Ergebnisse wurden sodann auf dem internationalen Gipfel in Quebec, Kanada im Juni 2002 zusammengetragen und verglichen (1). Ein viel versprechender Ansatz liegt in der Gestaltung des Tourismus in Naturschutzgebieten. 
(c) 20I0 Authors; licensee IÖW and oekom verlag. This is an article distributed under the terms of the Creative Commons Attribution Non-Commercial No Derivates License (http://creativecommons.org/licenses/by-nc-nd/3.o/), which permits unrestricted use, distribution, and reproduction in any medium, provided the original work is properly cited. 